\title{
Estado nutricional e carência de ferro em crianças freqüentadoras de creche antes e 15 meses após intervenção nutricional
}

\author{
Nutritional status and iron deficiency among children enrolled in a day care center before and after 15 \\ months of nutritional management
}

Terezinha Soares Biscegli', Carlos Elysio C. Corrêa ${ }^{2}$, João Romera ${ }^{3}$, André Binotti Candido ${ }^{4}$

\section{RESUMO}

Objetivo: Comparar o estado nutricional e a prevalência de carência de ferro em crianças freqüientadoras de creche antes e 15 meses após orientação nutricional e intervenção terapêutica.

Métodos: Estudo coorte com 52 crianças de 32 a 78 meses de idade, freqüentadoras de uma creche em Catanduva, São Paulo, avaliadas em dois períodos: antes e 15 meses após receberem tratamento para anemia e correção das alterações no estado nutricional. O estado nutricional das crianças foi avaliado por meio da curva da Organização Mundial de Saúde (2006). O diagnóstico de carência de ferro foi realizado por dosagem de hemoglobina e nível de ferritina sérica. Dados adicionais das crianças não eutróficas foram obtidos por meio de entrevistas com suas mães.

Resultados: Observou-se 2\% de desnutrição aguda e $8 \%$ de obesidade, não havendo diferença significante entre os dois períodos. $12 \%$ das crianças apresentaram anemia, havendo diminuição do número de crianças com anemia após o tratamento. Dois novos casos de obesidade e cinco de carência de ferro surgiram durante o período de intervenção nutricional. Das oito crianças com alterações nutricionais no período inicial, $63 \%$ das mães procuraram atendimento médico para tratamento.

Conclusões: O tratamento dos distúrbios nutricionais e da carência de ferro diminuiu o número de crianças afetadas. $\mathrm{O}$ aparecimento de novos casos de obesidade e anemia serve de alerta para a necessidade de outras medidas preventivas tanto na creche como no âmbito familiar.
Palavras-chave: criança; estado nutricional; anemia; anemia ferropriva.

\section{ABSTRACT}

Objective: To compare the nutritional status and the prevalence of iron deficiency among children enrolled in a day care center before and after 15 months of nutritional management.

Methods: Cohort study of 52 children, aged 32 to 78 months, evaluated before and after treatment of anemia and nutritional disturbs in a no-profit day care center in Catanduva, São Paulo, Brazil. The nutritional status was classified according to World Health Organization (2006) criteria. Iron deficiency anemia was detected by hemoglobin and ferritin levels. Additional characteristics of non-eutrophic children were obtained by interview with their mothers.

Results: Among the evaluated children, $2 \%$ presented acute malnutrition and $8 \%$ were obese, without significant difference between the two periods of the study. Abnormal values of hemoglobin and/or ferritin were noted in $12 \%$ of the children. During the study period, two new cases of obesity and five cases of iron deficiency were observed. Interview with the mothers of non-eutrophic children prior to intervention showed that $63 \%$ of them looked for medical assistance in order to treat the nutritional disorders.

Conclusions: The number of children with nutritional disorders and iron deficiency decreased. New cases of obesity and anemia indicate the need to adopt effective
1Pediatra, doutora em Medicina pela Faculdade de Medicina de Ribeirão Preto da Universidade de São Paulo (FMRP-USP), professora adjunta da Disciplina de Puericultura e Clínica Pediátrica do curso de Medicina das Faculdades Integradas Padre Albino (Fipa), Catanduva, SP, Brasil

2Pediatra, doutor em Medicina pela FMRP-USP, professor titular da Disciplina de Puericultura e Clínica Pediátrica do curso de Medicina da Fipa, Catanduva, SP, Brasil

${ }^{3}$ Pediatra, professor-assistente da Disciplina de Puericultura e Clínica Pediátrica do Curso de Medicina da Fipa, Catanduva, SP, Brasil

${ }^{4}$ Médico, residente de Pediatria do Hospital Padre Albino, Catanduva, SP, Brasil
Endereço para correspondência:

Terezinha Soares Biscegli

Rua Fortaleza, 95 - Jardim Ferreira

CEP 15840-000 - Itajobi/SP

E-mail: terezinhabiscegli@yahoo.com.br

Recebido em: 23/11/2007

Aprovado em: 29/1/2008 
preventive measures, both in the day care center and in the children's homes.

Key-words: child; nutritional status; anemia; anemia, iron-deficiency.

\section{Introdução}

A avaliação do estado nutricional é uma etapa fundamental no atendimento da criança e seu principal objetivo é verificar se o crescimento está se afastando do padrão esperado devido a alguma doença e/ou condições sociais desfavoráveis. O período entre o desmame e os cinco anos de idade é, nutricionalmente, a fase mais vulnerável do ciclo da vida ${ }^{(1)}$. Devido à alta prevalência de distúrbios, como a desnutrição e, mais recentemente, a obesidade, cuja incidência vem aumentando em nosso meio com grave prognóstico ${ }^{(2)}$, a vigilância nutricional constante faz-se necessária nos países em desenvolvimento ${ }^{(3)}$.

Dentre os fatores relacionados à gênese da desnutrição, destacam-se a desnutrição intra-uterina, a desnutrição pósnatal, a prematuridade, o abandono precoce do aleitamento materno, as infecções repetidas, além do fraco vínculo mãefilho ${ }^{(4)}$. Já no que concerne à obesidade, os principais fatores implicados estão estritamente relacionados às mudanças no estilo de vida (mais tempo em frente à televisão e jogos de computadores, maior dificuldade de brincar na rua pela falta de segurança) e nos hábitos alimentares (maior apelo comercial dos produtos ricos em carboidratos simples, gorduras e calorias, maior facilidade de fazer preparações ricas em gorduras e menor custo de produtos de padaria) ${ }^{(5)}$.

Outro grave problema de Saúde Pública em todo o mundo é a anemia carencial ferropriva, sendo $95 \%$ das anemias decorrentes da carência de ferro. Acontece com maior prevalência nos países em desenvolvimento, comprometendo principalmente a população materno-infantil ${ }^{(6)}$. No Brasil, pesquisadores têm encontrado substancial aumento na prevalência de anemia em crianças ao longo dos anos ${ }^{(7,8)}$. Pesquisas recentes mostraram prevalência de $69 \%$ em crianças de creche da rede municipal de São Paulo(o). A deficiência de ferro tem como conseqüências: perda da resistência às infecções, com infecções de repetição em curtos intervalos de tempo; redução da capacidade lúdica; perda de apetite e distúrbios na área neuropsicomotora - muitas vezes irreversíveis, mesmo após o tratamento adequado ${ }^{(3)}$.

Segundo a Lei de Diretrizes e Bases da Educação Nacional (Lei 9.394/96), creches são instituições que atendem a crianças de zero a três anos e pré-escolares de quatro a seis anos. As duas faixas etárias compreendem a educação infantil, que é a primeira etapa da educação básica e tem como finalidade o desenvolvimento integral da criança em seus aspectos físico, psicológico, intelectual e social ${ }^{(10)}$. A creche vem se tornando uma necessidade significativa da população, em conseqüência das transformações socioeconômicas ocorridas nas últimas décadas. Como as crianças permanecem na creche de oito a dez horas por dia e, durante este tempo, recebem dois terços de suas necessidades nutricionais, além da orientação psicopedagógica, é necessário que a alimentação e os cuidados oferecidos satisfaçam suas necessidades e influenciem favoravelmente seu estado nutricional e desenvolvimento neuropsicomotor ${ }^{(11)}$.

Em estudo desenvolvido anteriormente com as crianças freqüentadoras da creche Sinharinha Neto, situada no município de Catanduva, São Paulo ${ }^{(11)}$, detectou-se 3,9\% de desnutrição aguda, 1,9\% desnutrição crônica, $6,7 \%$ de obesidade e alta prevalência de carência de ferro (48\%). Durante um período de 15 meses, medidas terapêuticas e profiláticas foram adotadas, com o intuito de reduzir a prevalência destes distúrbios. O objetivo do presente estudo foi reavaliar o estado nutricional e a prevalência de carência de ferro no mesmo grupo de crianças, após o período de tratamento.

\section{Métodos}

Estudo coorte realizado entre setembro de 2005 e agosto de 2007. Foram estudadas 52 crianças, de 32 a 78 meses de idade, freqüentadoras da creche Sinharinha Neto no município de Catanduva, avaliadas antes e depois de tratamento para carência de ferro e distúrbios nutricionais durante um período de 15 meses. Todas as crianças foram classificadas como de baixa renda, de acordo com os critérios estabelecidos pela própria creche, que leva em consideração a renda familiar, o tipo de moradia, a composição familiar e a necessidade da mãe trabalhar fora de casa.

Determinou-se o estado nutricional e realizou-se a investigação de carência de ferro em todas as crianças selecionadas. Também foram realizadas entrevistas com as mães das crianças, com o objetivo de avaliar o desempenho das mesmas em cumprir com as obrigações a elas delegadas, ou seja, procurar o serviço médico indicado e realizar as condutas determinadas. Foram comparados os resultados anteriores $\left(\mathrm{T}_{1}\right)$ e os posteriores ao tratamento $\left(\mathrm{T}_{2}\right)$. 
A ficha de matrícula da criança foi utilizada para determinar a idade cronológica (em meses) e o sexo. A antropometria (peso e estatura) obedeceu as técnicas preconizadas pela Organização Mundial de Saúde (OMS) ${ }^{(12,13)}$ e foi realizada exclusivamente por um dos três autores, especialmente treinado para desempenhar tal tarefa. A medida do comprimento da criança de zero a 24 meses foi feita com o parente em decúbito dorsal (antropômetro horizontal) e, para os maiores de dois anos, aferiu-se a altura em posição ortostática (antropômetro vertical). A aferição do peso foi realizada com balança pediátrica (precisão de $10 \mathrm{~g}$ ) e balança plataforma (precisão de $100 \mathrm{~g}$ ), para crianças de até $15 \mathrm{~kg}$ ou acima de $15 \mathrm{~kg}$, respectivamente.

Para a avaliação nutricional, empregaram-se as novas curvas da OMS (2006) $)^{(14)}$, que levam em conta as relações entre peso/altura $(\mathrm{P} / \mathrm{A})$ e altura/idade $(\mathrm{A} / \mathrm{I})$, sendo o escore $\mathrm{Z}_{\leq-2} \mathrm{e} \geq+2$ utilizados para definir desnutrição e obesidade, respectivamente. Foram consideradas eutróficas as crianças que apresentaram relação $\mathrm{P} / \mathrm{A}$ e $\mathrm{A} / \mathrm{I}$ normais; com desnutrição aguda as que apresentaram P/A abaixo do limite e A/I normal; e com desnutrição pregressa as que apresentaram P/A normal e A/I abaixo do limite. Foram classificadas como obesas as crianças que apresentaram P/A acima do limite pré-estabelecido.

O diagnóstico de carência de ferro foi feito com base nas dosagens sangüíneas de hemoglobina (Coulter T-890) e ferritina (quimioluminescência). Foram determinados os seguintes pontos de corte: hemoglobina $<11 \mathrm{~g} / \mathrm{dL}$ (em crianças menores de 60 meses) e $<12 \mathrm{~g} / \mathrm{dL}$ (em crianças maiores de 60 meses) ${ }^{(15)}$ e ferritina $<10 \mathrm{ng} / \mathrm{mL}$. Considerou-se a presença da carência de ferro quando os valores de hemoglobina e/ou ferritina estavam abaixo dos valores de corte.

Após avaliação da reserva de ferro, as crianças receberam dois esquemas de intervenção. A abordagem preventiva foi aplicada em 27 crianças que não eram portadoras de carência de ferro e a abordagem terapêutica, em 25 crianças que apresentavam valores baixos de hemoglobina e/ou ferritina. O esquema terapêutico foi feito com sulfato ferroso na dose de $3 \mathrm{mg} / \mathrm{kg} / \mathrm{dia}^{(16)}$ e o profilático com $1 \mathrm{mg} / \mathrm{kg} / \mathrm{dia}^{(16)}$, por um período de três meses. A administração do medicamento ficou sob a responsabilidade das funcionárias da creche. As oito crianças com distúrbios nutricionais em $\mathrm{T}_{1}$ (obesidade ou desnutrição) foram encaminhadas para consultas médicas em ambulatório especializado, sendo responsabilidade das mães seguir o tratamento recomendado, já que não havia possibilidade de personalizar as dietas na própria creche.

O presente projeto de pesquisa foi aprovado pelo Comitê de Ética da Faculdade de Medicina de Catanduva e foi solicitada a assinatura do termo de consentimento livre e esclarecido pelos responsáveis legais das crianças.

Os resultados foram expressos em porcentagem, média e desvio padrão. Para a análise das variáveis, utilizou-se o teste $\mathrm{Z}$ para duas proporções. Considerou-se significante $p<0,05$.

\section{Resultados}

Das 52 crianças estudadas, 22 (42\%) eram do sexo masculino e $30(58 \%)$ do feminino. A idade das crianças variou

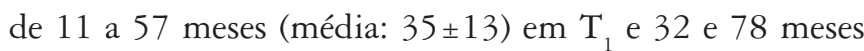
(média: $56 \pm 13$ ) em $\mathrm{T}_{2}$. A faixa etária predominante foi a de 25 a 60 meses tanto em $\mathrm{T}_{1}$ (39 crianças, $75 \%$ ) como em $\mathrm{T}_{2}$ (34 crianças, 65\%).

A avaliação do estado nutricional está demonstrada na Tabela 1. Das quatro crianças obesas observadas em $T_{1}$, duas evoluíram para eutrofia e duas permaneceram obesas. Dois novos casos de obesidade foram detectados em $\mathrm{T}_{2}$. Das duas crianças com desnutrição pregressa em $\mathrm{T}_{1}$, uma evoluiu para eutrofia e a outra para desnutrição aguda. As duas crianças com desnutrição aguda em $T_{1}$ tornaram-se eutróficas em $T_{2}$. A desnutrição aguda incidiu apenas na faixa etária de 25 a 60 meses, detectando-se três $(6 \%)$ crianças nessa condição. Observou-se apenas um (2\%) caso de desnutrição pregressa em menores de dois anos e um (2\%) caso entre crianças de 25 a 60 meses. A obesidade predominou nos maiores de 24 meses (principalmente nos maiores de 60 meses), com sete

Tabela 1 - Distribuição das crianças de acordo com o estado nutricional nos tempos $T_{1}$ e $T_{2}$

\begin{tabular}{lcrcrl}
\hline Estado nutricional & \multicolumn{2}{c}{$\mathrm{T}_{1}$} & \multicolumn{2}{c}{$\mathrm{T}_{2}$} & \multirow{2}{*}{$\begin{array}{c}\text { Número de } \\
\text { casos }\end{array}$} \\
\cline { 2 - 5 } & casos & $\%$ & 47 & 90 & 0,37 \\
\hline Eutrofia & 44 & 85 & 1 & 2 & 0,56 \\
Desnutrição aguda & 2 & 4 & 0 & 0 & 0,15 \\
Desnutrição pregressa & 2 & 4 & 4 & 8 & 0,50 \\
Obesidade & 4 & 7 & $\mathbf{5 2}$ & $\mathbf{1 0 0}$ & \\
Total & $\mathbf{5 2}$ & $\mathbf{1 0 0}$ & &
\end{tabular}


(14\%) crianças. Nenhum caso de obesidade foi observado em menores de dois anos. A proporção obesidade/desnutrição em $T_{1}$ foi de $1 / 1$, enquanto que em $T_{2}$ foi de $4 / 1$. Esta diferença foi significante $(p<0,001)$. No que se refere ao sexo, os distúrbios nutricionais predominaram no sexo feminino, com sete (14\%) casos em meninas e quatro (8\%) em meninos.

As entrevistas com as mães das oito crianças que apresentavam distúrbios nutricionais em $\mathrm{T}_{1}$ revelaram que cinco delas (63\%) procuraram o atendimento médico recomendado e seguiram as orientações dietéticas até a recuperação do estado nutricional e três (38\%) não demonstraram interesse em fazê-lo.

A distribuição dos valores séricos de hemoglobina e ferritina está demonstrada na Tabela 2. Das 25 crianças que apresentaram carência de ferro em $\mathrm{T}_{1}, 24$ (96\%) tiveram seus exames normalizados em $\mathrm{T}_{2}$. Cinco crianças (10\%) que não demonstraram carência em $\mathrm{T}_{1}$ desenvolveram anemia em $\mathrm{T}_{2}$.

\section{Discussão}

A análise dos dados antropométricos das crianças freqüentadoras da creche Sinharinha Neto após o período de 15 meses de intervenção nutricional revelou redução significante $(p<0,05)$ do número de desnutridos (de $8 \%$ para $2 \%$ ), embora o número de obesos tenha permanecido o mesmo (8\%). Consequentemente, a proporção de obesos/desnutridos modificou-se significativamente (de $1 / 1$ para 4/1), evidenciando mudança do padrão nutricional. Resultado diferente foi observado em estudo desenvolvido em creches beneficentes do município de São Paulo, que demonstrou prevalência similar de desnutrição $(7 \%)$ e obesidade (6\%) (17). Outra pesquisa com crianças de creche da cidade de Catanduva $^{(18)}$ também evidenciou maior número de obesos (16\%), comparado ao número de desnutridos (11\%), embora a prevalência de distúrbios nutricionais tenha sido maior que a da presente pesquisa. Corso et a ${ }^{(19)}$ avaliaram crianças menores de seis anos na cidade de Florianópolis, Santa Catarina, e também demonstraram maior prevalência de sobrepeso/obesidade $(6,8 \%)$ comparada à de desnutrição aguda (2\%). Já outra pesquisa desenvolvida com crianças de ambulatório pediátrico detectou uma porcentagem maior de desnutridos $(27 \%)$ comparada à de obesos $(14,5 \%)^{(20)}$. Tal divergência de resultados possivelmente se explica pelo fato de se tratarem de populações diversas, avaliadas por critérios diferentes.

Nos últimos 30 anos, houve uma mudança nos padrões nutricionais da população brasileira (transição nutricional), com evidente diminuição de desnutridos e aumento de sobrepeso ou obesidade, principalmente devido aos hábitos alimentares inadequados ${ }^{(21)}$. Este aumento de obesidade é observado em todas as camadas da população, mas, principalmente, em famílias de menor poder aquisitivo ${ }^{(22)}$. Os resultados de nossa pesquisa concordam com os achados destes autores ${ }^{(21,22)}$. Vale lembrar que, embora nas últimas décadas esteja ocorrendo redução da prevalência de desnutrição calórico-protéica em crianças menores de cinco anos no Brasil, esta é ainda considerada um problema de Saúde Pública, principalmente nas regiões Nordeste e Norte, nos denominados bolsões de pobreza ${ }^{(23)}$.

Ao comparar os dados das crianças com distúrbios nutricionais em $\mathrm{T}_{1}$ e em $\mathrm{T}_{2}$ (Tabela 1 ), não houve diferença significante entre os dois períodos (oito e cinco crianças, respectivamente), embora as crianças do primeiro período tivessem sido encaminhadas para atendimento especializado. Tal achado poderia ser explicado pelo fato de que somente $63 \%$ das mães seguiram as recomendações e realizaram o tratamento ou porque durante os dois estudos surgiram dois novos casos de obesidade.

Crianças freqüentadoras de creche obtêm praticamente $70 \%$ de suas necessidades nutricionais durante o período de permanência nessas instituições e recebem alimentação balanceada durante praticamente 10 horas do dia, com quantidades suficientes de nutrientes. Tais crianças deveriam estar mais protegidas de distúrbios nutricionais e anemias carenciais, comparadas às crianças da população geral $^{(24,25)}$. A alimentação oferecida às crianças aqui estudadas foi preparada pela cozinha da Prefeitura Municipal, que obedecia a critérios estabelecidos por nutricionista. Tratavase de dieta geral, adequada para as diferentes faixas etárias,

Tabela 2 - Distribuição das crianças segundo valores séricos de hemoglobina $(\mathrm{Hb})$ e ferritina nos tempos $\mathrm{T}_{1}$ e $\mathrm{T}_{2}$

\begin{tabular}{|c|c|c|c|c|c|}
\hline \multirow[b]{2}{*}{ Valores séricos } & \multicolumn{2}{|c|}{$T_{1}$} & \multicolumn{2}{|c|}{$T_{2}$} & \multirow[b]{2}{*}{$p$} \\
\hline & $\begin{array}{l}\text { Número de } \\
\text { casos }\end{array}$ & $\%$ & $\begin{array}{l}\text { Número de } \\
\text { casos }\end{array}$ & $\%$ & \\
\hline $\mathrm{Hb}<11^{*}$ ou $<12^{* *} \mathrm{~g} / \mathrm{dL}$ & 20 & 39 & 6 & 12 & $<0,001$ \\
\hline Ferritina <10ng/mL & 11 & 21 & 0 & 0 & $<0,001$ \\
\hline $\mathrm{Hb}$ e/ou Ferritina baixos & 25 & 48 & 6 & 12 & $<0,001$ \\
\hline
\end{tabular}

*para crianças entre zero e 60 meses de idade $\mathrm{e}^{* *}$ para crianças $>60$ meses. 
oferecida em horários controlados. Dessa forma, o fato de terem surgido três novos casos de obesidade alerta para a possibilidade de que os fatores desencadeantes estejam relacionados ao ambiente familiar (após a saída da creche, nos finais de semana, feriados e férias), já que, na creche, as transgressões alimentares são pouco prováveis. Outro fator que poderia contribuir para o aumento do número de obesos é que, na creche, os exercícios físicos geralmente são bastante leves, pois não existe espaço físico amplo para desenvolver atividades aeróbicas, tais como correr, jogar bola, andar de bicicleta e praticar natação.

A anemia na infância está relacionada a deficiências nas áreas sociais, econômicas e culturais. A deficiência de ferro é a carência nutricional mais prevalente no mundo, embora sua prevenção seja bem estabelecida. A OMS estima que, nos países em desenvolvimento, mais de $50 \%$ das crianças menores de quatro anos apresentem deficiência de ferro. A principal causa da anemia ferropriva, nestes países, está ligada à ingestão alimentar insuficiente de ferro ${ }^{(11)}$.

A reavaliação de carência de ferro das crianças freqüentadoras da creche Sinharinha Neto, após o período de 15 meses (Tabela 2), revelou redução significativa na prevalência de anemia. Tal resultado foi conseguido por meio de medidas simples e de baixo custo, colocadas em prática na própria creche. A administração do sulfato ferroso pelas funcionárias durante um período de três meses mostrou-se altamente vantajosa não só para as crianças, mas também para as mães, que foram aliviadas de alguns cuidados com relação aos seus filhos, haja vista a jornada dupla de trabalho (fora e dentro do lar) a que são submetidas. Embora o tratamento das crianças que apresentaram carência em $\mathrm{T}_{1}$ tenha sido altamente eficaz (96\% de êxito), verificou-se cinco novos casos à época da reavaliação. Tais crianças desenvolveram anemia, apesar de terem recebido tratamento profilático medicamentoso por um período de três meses, sugerindo que tal medida foi insuficiente para prevenir o distúrbio.

A OMS propõe que o controle de anemia ferropriva seja efetuada por meio de educação alimentar, controle de infecções parasitárias, suplementação medicamentosa e fortificação de alimentos com sais de ferro ${ }^{(3)}$. Sabe-se que a quantidade adequada de ferro na dieta é a principal responsável pela reserva de ferro e, portanto, dietas bem balanceadas contribuem para a reduzir a prevalência de anemia. Como a dieta fornecida pela creche é balanceada e, teoricamente, deveria suprir $70 \%$ das necessidades individuais, provavelmente a contrapartida do domicílio não é suficiente para a demanda global.

$\mathrm{O}$ combate à carência de ferro com medidas eficazes em curto prazo, tal como a suplementação periódica e sistemática com sais de ferro, seja na forma medicamentosa ou na forma de alimentos enriquecidos, já vem sendo realizada com sucesso em alguns locais ${ }^{(26)}$. Vitolo $e t$ al ${ }^{(27)}$ evidenciaram redução da carência de ferro ao introduzir cereais enriquecidos com ferro na dieta habitual das crianças em idade pré-escolar. Pesquisa realizada por Torres et al ${ }^{(28)}$ também demonstrou que a prevalência de anemia pode ser reduzida com medidas simples, como fortificar o leite com ferro e vitamina C. Almeida et al ${ }^{(29)}$, em estudo desenvolvido em Pontal, São Paulo, demonstraram que o suco de laranja enriquecido com ferro, incorporado ao cardápio da creche, pode contribuir para a recuperação de crianças com deficiência de ferro e prevenir a deficiência em outras crianças. Estudos realizados em crianças freqüentadoras de creches públicas e filantrópicas do município de São Paulo concluíram que a elevada prevalência de anemia na população estudada caracteriza a situação como um grave problema de Saúde Pública, com necessidade urgente de instituir medidas efetivas para seu combate e prevenção( ${ }^{(17,30)}$.

De acordo com os resultados observados no presente estudo, verifica-se que a prevalência de desnutrição está em franca redução, mas que a prevalência de obesidade está aumentando na população de baixa renda ${ }^{(22)}$. Outro fator digno de nota é a persistência de casos de anemia em crianças que receberam tratamento curativo e profilático. Pode-se concluir que o aparecimento de novos casos de obesidade e anemia em uma população de crianças sob vigilância e tratamento específicos indica a necessidade de se adotar outras medidas preventivas, tanto na creche como no âmbito familiar. A fortificação de alimentos como cereais, leite e sucos poderia ser adotada pela creche, assim como a prática de atividades aeróbicas. Seria benéfico fornecer orientação nutricional às mães, por meio de palestras por pessoal capacitado, ensinando e estimulando hábitos alimentares saudáveis e incentivando a prática de esportes durante os finais de semana. $\mathrm{O}$ acompanhamento médico e a vigilância nutricional realizada por pessoal especializado na própria creche seriam de grande valia para detectar e intervir precocemente nos casos de distúrbios. Dessa forma, creche e família, atuando de maneira interativa, poderiam contribuir para promover a saúde infantil.

\section{Agradecimentos}

À coordenadora da creche Sinharinha Neto (Sra. Maria Helena Denadai), à recepcionista do Hospital Emílio Carlos (Srta. Margarida Marioti) e às alunas da Liga de Pediatria (Cibele C. Castilho, Gabriela R. Donadeli, Maria Paula G. da Silva e Mariana B. Tristão) pelo auxílio na parte operacional.Aos professores, funcionários, pais e crianças, pela participação e interesse. 


\section{Referências bibliográficas}

1. Monte C. Malnutrition: a secular challenge to child nutrition. J Pediatr (Rio J) 2000;76 (Suppl 3):S285-97.

2. Comitê de Nutrição da Sociedade de Pediatria de São Paulo. Avaliação do estado nutricional das crianças. Rev Paul Pediatr 1992;10:102-3.

3. Unicef/UNU/WHO/MI, editors. Preventing iron deficiency in women and children: background and consensus on key technical issues and resources for advocacy, planning and implementing national programs [technical workshop on the Internet]. New York: Internacional Nutrition Foundation and Micronutrient Initiative; 1998 [cited 2007 Nov 5]. Available from: http://www.inffoundation. org/pdf/prevent_iron_def.pdf

4. Nóbrega FJ, Campos ALR, Nascimento CFL. Vínculo mãe/filho na desnutrição. In: Nóbrega FJ, Campos ALR, Nascimento CFL, editores. Distúrbios nutricionais e fraco vínculo mãe/filho. 2 ed. Rio de Janeiro: Revinter; 2000. p. 55-65.

5. Wang Y, Monteiro C, Popkin BM. Trends of obesity and underweight in older children and adolescents in the United States, Brazil, China, and Russia. Am J Clin Nutr 2002;75:971-7.

6. Dallman PR, Siimes MA, Stekel A. Iron deficiency in infancy and childhood. Am J Clin Nutr 1980;33:86-118.

7. Monteiro CA, Szarfarc SC, Mondini L. Secular trends in childhood in the city of São Paulo, Brazil (1984-1996). Rev Saude Publica 2000;34:62-72.

8. Torres MA, Sato K, Queiroz $S$ de S. Anaemia in children under two years in basic health care units in the State of São Paulo, Brasil. Rev Saude Publica 1994;28:290-4.

9. Bueno MB, Selem SS, Areas JA, Fisberg RM. Prevalência e fatores associados à anemia entre crianças atendidas em creches públicas de São Paulo. Rev Bras Epidemiol 2006;9:462-70.

10. Brasil - Ministério da Educação [homepage on the Internet]. Diretrizes e bases da educação nacional: Lei nº. 9.394, 1996 [cited 2007 Feb 6]. Available from: http://www.planalto.gov.br/ccivil_03/Leis/L9394.htm

11. Biscegli TS, Corrêa CE, Romera J, Hernandez JL. Avaliação do estado nutricional e prevalência de carência de ferro em crianças freqüentadoras de uma creche. Rev Paul Pediatr 2006;24:323-9.

12. Autoria não referida. Physical status: the use and interpretation of anthropometry. Report of a WHO Expert Committee. World Health Org Tech Rep 1995;854:1-452.

13. Hamill PV, Drizd TA, Johnson CL, Reed RB, Roche AF. NCHS growth curves for children birth-18 years. United States. Vital Health Stat 1977;11:1-74.

14. de Onis M, Garza C, Victora CG, Onyango AW, Frongillo EA, Martines J. The WHO Multicentre Growth Reference Study: planning, study design, and methodology. Food Nutr Bull 2004;25 (1 Suppl):S15-26.

15. Organización Mundial de la Salud [homepage on the Internet]. Lucha contra la anemia nutricional específicamente contra la carencia de hierro [Serie de Informes Técnicos, 580]. Ginebra: OMS; 1975 [cited 2007 Nov 5]. Available from : http://whqlibdoc.who.int/trs/WHO_TRS_580_spa.pdf
16. Autoria não referida. Recommendations to prevent and control iron deficiency in the United States. Centers for Disease Control and Prevention. MMWR Recomm Rep 1998;47:1-29.

17. Machado EH, Brasil AL, Palma D, Taddei JA. Condição nutricional e prevalência de anemia em crianças matriculadas em creches beneficentes. Rev Paul Pediatr 2005;23:21-6.

18. Biscegli TS, Polis LB, Santos LM, Vicentin M. Avaliação do estado nutricional e do desenvolvimento neuropsicomotor em crianças freqüentadoras de creche. Rev Paul Pediatr 2007;25:337-42.

19. Corso AC, Botelho JL, Zeni LA, Moreira EA. Sobrepeso em crianças menores de seis anos de idade em Florianópolis, SC. Rev Nutr 2003;16:21-8.

20. Torres MA, Queiroz SS, Schvartsman C, Santos E, Nóbrega FJ. Prevalência de anemia e avaliação da condição nutricional de crianças atendidas no Ambulatório de Pediatria do Programa Einstein na Comunidade de Paraisópolis. Rev Paul Pediatr 2002;20:61-8.

21. Batista Filho M, Rissin A. Nutritional transition in Brazil: geographic and temporal trends. Cad Saude Publica 2003;19 (Suppl 1):S181-91.

22. Taddei JA, Colugnati FA, Rodrigues EM. Transição nutricional em menores de 5 anos: evidência dos inquéritos antropométricos brasileiros. In: Cardoso AL, Lopes LA, Taddei JA (editores). Tópicos atuais em nutrição pediátrica. São Paulo: Atheneu; 2004. p. 11-43.

23. Monteiro CA, Conde WL. Secular trends in malnutrition and obesity among children in the city of São Paulo, Brazil (1974-1996). Rev Saude Publica 2000;34:52-61.

24. Silva LS, Giuglian ER, Aerts DR. Prevalence and risk factors for anemia among children in Brazil. Rev Saude Publica 2001;35:66-73.

25. Taddei JA, Cannon MJ, Warner L, Souza P, Vitalle S, Palma D et al. Nutritional gains of underprivileged children attending a day care center in Sao Paulo City, Brazil: a nine month follow-up study. Rev Bras Epidemiol 2000;3:29-37.

26. Ferraz IS, Daneluzzi JC, Vannucchi H, Jordão AA Jr, Ricco RG, Del Ciampo LA et al. Prevalence of iron deficiency and its association with vitamin A deficiency in preschool children. J Pediatr (Rio J) 2005;81:169-74.

27. Vitolo MR, Aguirre AN, Kondo MR, Juliano Y, Ferreira M, Lopes FA. Impacto do uso de cereal adicionado de ferro sobre os níveis de hemoglobina e a antropometria de pré-escolares. Rev Nutr 1998;11:163-71.

28. Torres MA, Sato K, Lobo NF, de Souza Queiroz S. The effect of the use of milk fortified with iron and vitamin $\mathrm{C}$ on hemoglobin levels and nutritional status of children under 2. Rev Saude Publica 1995;29:301-7.

29. Almeida CA, Ricco RG, Ciampo LA, Souza AM, Pinho AP, Oliveira JE. Factors associated with iron deficiency anemia in Brazilian preschool children. $J$ Pediatr (Rio J) 2004;80:229-34.

30. Ling NT, Ribeiro LC, Konstantyner T, Taddei JA. Anemia em lactentes que freqüentam creches públicas e filantrópicas no Município de São Paulo - SP. Rev Paul Pediatr 2006;24:330-4. 